\title{
Absence of immediate transfer of training of duration symbolic-matching-to-sample in pigeons
}

\author{
PHILIPP J. KRAEMER \\ University of Kentucky, Lexington, Kentucky
}

\begin{abstract}
Two experiments examined the performance of pigeons on symbolic-matching-to sample in which the relevant sample dimension consisted of duration. Each pigeon was trained on two problems that had the same two sample durations, 2 and $10 \mathrm{sec}$, but were different with respect to other physical properties of the samples. Durations of light and tone were used in Experiment 1; durations of two different color-location compounds were used in Experiment 2. In each experiment, a unique choice stimulus was associated with each of the four possible combinations of duration and signal type. Test sessions contained probe trials in which the choice stimuli were those appropriate for a long and a short duration of the signal type opposite to that actually presented. Pigeons in both experiments displayed asymmetrical performance deficits. Accuracy on long durations dropped to chance or below, whereas accuracy on short durations remained high. This pattern is similar to the choose-short effect that is obtained when animals are tested with long retention intervals. The implications of these results for duration memory, coding, and transfer of training are discussed.
\end{abstract}

There now exist many documented examples of the ability of animals to perceive and discriminate stimulus duration (Church, 1978; DeLong \& Wasserman, 1985; Gibbon \& Allan, 1984; Killeen \& Fetterman, 1988; Richelle \& Lejeune, 1980). In some situations, this ability has appeared as a tendency to process stimulus duration independently of other sensory-specific attributes. For example, rats trained to respond to one lever after a short duration light and a different lever after a long duration light, can transfer the discrimination to short and long duration tones (Church \& Meck, 1984; S. Roberts, 1982). Pigeons also show cross-modal transfer of a duration discrimination, although in a more constrained fashion than do rats. W. A. Roberts, Cheng, and Cohen (1989) found immediate positive transfer in a group of pigeons trained with tone durations and then tested with light durations, but transfer did not occur in another group of birds trained with these signals in the reverse order (light to tone).

Transfer of duration discriminations is not just a sensory modality effect-it also seems to occur with different signals from the same modality. As an example, Spetch and Sinha (1989) trained pigeons with a symbolic-matchingto-sample (SMTS) procedure in which the birds learned to choose a correct color stimulus as a function of the duration of one of two different visual signals. In a subsequent test phase, two signal durations appeared in succession, separated by various intervals, and the birds behaved

This research was supported by a grant from the National Institute of Mental Health (1R01 MH44082-01) to Philipp J. Kraemer. I wish to thank David McKinzie for his assistance in completing this project and Peter Urcuioli for his helpful insights on avian pedagogy. Correspondence should be addressed to the author at the Department of Psychology, Kastle Hall, University of Kentucky, Lexington, KY 40506-0044. as if they were adding the two durations. Interestingly, this effect occurred both when the same signal type occurred twice and when two different signal types appeared.

A common feature in all of these studies is that the same response alternatives were used with both signal types. For example, W. A. Roberts et al. (1989) used a procedure in which a left or a right choice response was contingent on the duration of a preceding tone; the same response alternatives were then available during transfer training with light durations, and the same response rules were in effect-for example, "respond to the left key if preceded by a short duration signal." Similarly, the procedure used by Spetch and Sinha (1989) held constant the response alternatives (red and green keylights) and the response rules associated with durations of the two visual samples. The same is also true of studies with rats that have involved spatial choice responses (Church \& Meck, 1984).

The goal of the present study was to examine how pigeons would perform when different sets of response alternatives were correlated with the same durations of two different signal types. A symbolic-matching-to-sample procedure was used in which pigeons were trained to respond to two different sample types. In Experiment 1, the samples were short and long lights and tones; in Experiment 2 the samples were short and long durations of two different visual signals. The important aspect of training was that two different sets of choice stimuli were correlated with the different signal types. For example, a subject might be trained to respond to red following a 2-sec light, to blue following a 10-sec light, to yellow following a 2-sec tone, and to green following a 10-sec tone. Throughout acquisition, red and blue would always follow a light duration, and yellow and green would always follow a tone duration. The critical question was how the 
pigeons would perform when tested with a set of choice alternatives that were not correlated with the preceding sample-for example, when after a 2- or 10-sec light, yellow and green would appear, instead of red and blue. If sample duration is all that matters, then pigeons should do well regardless of the set of choice alternatives presented, as long as a color is available that correlates with the duration of the preceding sample. This result would be consistent with transfer studies that have shown that animals can equate the durations of stimuli even when those stimuli differ with respect to other properties. Alternatively, pigeons trained with separate sets of choice stimuli may not readily abstract signal duration. This possibility would be especially likely if pigeons represent each trial of duration SMTS prospectively; if the birds code each trial in terms of a specific choice stimulus associated with a given sample, then they would not have available the information necessary to respond to choice stimuli associated with a sample type not presented (Kraemer, Mazmanian, \& Roberts, 1985). Thus, the outcome of these experiments is not only relevant to the study of temporal processing and transfer of training, but the results may also be pertinent to important issues related to coding and event representation.

\section{EXPERIMENT 1}

Pigeons were trained on two conditional discriminations. One involved presentations of light for 2 or $10 \mathrm{sec}$, followed by two color choice alternatives. The other problem involved 2- and 10-sec presentations of a tone, followed by a different set of color alternatives. Following the acquisition of both problems, test sessions were introduced that contained randomly arranged presentations of the four possible sample types (2- or 10-sec durations of light or tone), followed on nonprobe trials by the appropriate set of choice alternatives and on probe trials by the two choice stimuli that were appropriate to the signal type opposite to that presented.

\section{Method}

Subjects and Apparatus. Four White Carneaux pigeons, obtained from a commercial breeder, served as subjects. Each bird was individually housed in a stainless steel cage located in an environmentally controlled vivarium that operated on a 12:12-h light:dark cycle; all testing occurred during the light-on period. Subjects were maintained at $85 \%$ of their free-feeding weights, and they were weighed and fed after each daily session. Testing occurred 6 days each week. Grit and water were always available. The apparatus consisted of a standard sound-attenuated test chamber for pigeons. Three horizontally aligned plastic pecking keys, each fitted with a multistimulus projector, were mounted on the front wall. A modified houselight was mounted in the center of the ceiling and was fitted with a $4 \times 5 \mathrm{~cm}$ white translucent plastic cover. The center pecking key was covered with a convex plastic lens, which provided diffuse illumination similar to the ceiling light; it was used to present a red light in Experiment 2. A speaker was located in the lower left corner of the front wall. A $5 \times 6 \mathrm{~cm}$ opening located in the lower center of the front wall was used to present mixed grain through a electromechanical food hopper. The food access area was illuminated with white light only when food was available. The choice stimuli consisted of red, blue, green, and yellow solid colors presented on the side keys. The auditory sample was a $1000-\mathrm{Hz}, 84$ $\mathrm{dB}$ tone; the visual sample involved illumination of the .1-A, 28-V ceiling light (luminance $=8,200 \mathrm{~cd} / \mathrm{m}^{2}$ ). All trial-event programming and response recording were controlled by a Commodore 64 computer and a locally constructed computer interface.

Procedure. Each bird was initially hand-shaped to peck a whiteilluminated side key for a 2-sec access to mixed grain. Once responding was established, four preliminary training sessions were conducted. Each session contained $\mathbf{4 8}$ trials, and each trial involved presentation of white light on either the right or the left side key; spatial location of the illuminated key was balanced across sessions and randomized over trials within a session. A single peck to an illuminated side key produced a 4-sec access to grain, followed by a 15-sec intertrial interval (ITI). The chamber remained dark during the ITI. Responses to a dark side key had no effect. All birds completed four preliminary training sessions prior to task acquisition, at which time they were randomly assigned to one of two training conditions.

The original design counterbalanced task acquisition, so that two birds were to be trained first on the visual problem and next on the auditory problem; the other two birds were to be trained on these problems in the reverse order. This plan was aborted, however, after it became apparent that the two birds being trained on the auditory problem were not progressing. Consequently, a revised training procedure was initiated. All four pigeons were trained on the visual problem, followed by modified training on the auditory problem. The procedure used to train visual discrimination involved daily sessions composed of $\mathbf{4 8}$ trials. An equal number of short and long light presentations were randomly arranged within a session, and the left/right orientation of the choice stimuli was balanced and randomized across trials. The pairings of samples and correct choice stimuli for each subject are presented in Table 1.

Each trial began with a light sample that lasted for 2 or $10 \mathrm{sec}$, followed immediately by the choice stimuli. A single peck to either side key terminated the choice stimuli and either produced a 3-sec access to grain following a correct response or initiated a 15-sec ITI following an incorrect response; on correct trials, the ITI began immediately after food presentation. The chamber remained dark except for presentations of the sample of choice stimuli. Training continued until the subject achieved $85 \%$ correct responses during five consecutive sessions, at which time the bird was transferred to the auditory problem.

Auditory training involved several different procedures, given that the standard procedure used for the visual problem proved to

Table 1

Sample- and Correct-Choice Arrangements in Experiment 1

\begin{tabular}{cccl}
\hline Subject & Signal Type & $\begin{array}{c}\text { Sample Duration } \\
\text { (in sec) }\end{array}$ & $\begin{array}{c}\text { Correct-Choice } \\
\text { Stimulus }\end{array}$ \\
\hline 1 & Light & 2 & Red \\
& & 10 & Blue \\
& Tone & 2 & Green \\
4 & Light & 10 & Yellow \\
& & 2 & Blue \\
& Tone & 10 & Red \\
& & 2 & Yellow \\
2 & Light & 10 & Green \\
& & 2 & Green \\
& Tone & 10 & Yellow \\
8 & & 2 & Red \\
& Light & 10 & Blue \\
& & 2 & Yellow \\
& Tone & 10 & Green \\
& & 2 & Blue \\
& & 10 & Red \\
\hline
\end{tabular}


be unsuccessful when it was used with auditory stimuli. The first strategy involved trial blocking, in which the same signal duration occurred during the first 24 trials of a session, followed by 24 trials of the other duration; this continued for about 50 sessions for each bird and was unsuccessful. The next strategy involved a correction procedure that entailed presentation of the choice stimuli until a correct response occurred; responses to the incorrect stimulus had no effect. This procedure was also ineffective. The final strategy, which was successful, involved a different kind of correction procedure. A response to either side key ended the trial and was followed by reinforcement and the ITI after correct choices and just the ITI after incorrect choices. Incorrect responses, however, produced a repetition of the previous trial type, which continued until a correct response occurred. Once a pigeon reached the criterion of $85 \%$ correct or better on 5 consecutive sessions, the correction procedure was omitted and an additional 5 sessions were completed. This phase was followed by 10 mixed-sample sessions. These 48-trial sessions contained an equal number of short and long tones and short and long lights; each sample was followed by the appropriate choice-stimulus set (see Table 1), with the left/right arrangement of the choice stimuli balanced across trials. The four sample types ( 2 durations $\times 2$ modalities) were presented in a different random order for each subject and for each session; reinforcement following correct responses was reduced to $2 \mathrm{sec}$. Ten mixedsample sessions were presented, followed by the test phase.

Test sessions were similar to the mixed-sample sessions, except that $\mathbf{1 6}$ probe trials were included. Each of the four possible combinations of sample modality (auditory or visual) and duration (2 or $10 \mathrm{sec}$ ) were presented four times each as probe trials. The 16 probe trials were randomly intermixed among 40 nonprobe trials, resulting in $\mathbf{5 6}$ trials per session. The nonprobe trials were of the same type as those presented during the preceding training phase. Probe trials differed from nonprobe trials in two respects: First, the choice-stimulus set was the one appropriate for the sample modality opposite to that actually presented (see Table 1). Second, reinforcement did not occur on probe trials. Test sessions alternated regularly over days with standard mixed-trial sessions, until a total of 12 test sessions were completed.

\section{Results and Discussion}

All statistical analyses were evaluated with a significance level set at $p<.05$. There is no doubt that the pigeons found the auditory problem more difficult to acquire than the visual problem. Mean sessions to criterion on the auditory problem was 228 , whereas mean sessions to criterion on the visual problem was 46 . The modified training procedure required with the auditory problem prevents any valid statistical comparison of the rates of acquisition, but the magnitude of the difference nonetheless captures the relative difficulty of the two problems. Consistent with other studies of SMTS in pigeons, acquisition was much easier with visual than with auditory samples (Kraemer \& Roberts, 1984; W. A. Roberts, Cheng, \& Cohen, 1989).

The primary data of interest are the scores from the test sessions in which probe trials were inserted. Correct responses were defined for probe and nonprobe trials with respect to the choice stimulus that was correct for the duration of the sample presented, irrespective of sample modality. Accuracy remained high on nonprobe trials throughout testing ( $M=87 \%$ ), but performance dropped significantly on probe trials $(M=48 \%)$. A more detailed examination of these data, however, revealed that the pat-
Table 2

Percent Correct on Nonprobe and Probe Trials in Experiment 1

\begin{tabular}{ccccc}
\hline \multicolumn{2}{c}{ Nonprobe Trials } & & \multicolumn{2}{c}{ Probe Trials } \\
\cline { 4 - 5 } \cline { 4 - 5 } Short & Long & & Short & Long \\
\hline 82 & 85 & & 72 & 21 \\
\hline
\end{tabular}

tern of performance was actually more complicated. Table 2 contains the mean percent correct choices for short and long samples, collapsed over sample modality, for probe and nonprobe trials. A mixed factorial analysis of variance (ANOVA) was performed on percent correct scores, with group (Subjects 1 and 4 vs. Subjects 2 and 8 ) as the between-groups factor; the within-subjects factors consisted of trial type (probe or nonprobe), sample modality (auditory or visual), test block (three blocks of four sessions each), and signal duration ( 2 or $10 \mathrm{sec})$. The main effects of signal duration and trial type reached significance $[F(2,2)=23.1$ and $F(2,2)=112.0$, respectively]. The interaction between these factors was also significant $[F(2,2)=22.4]$, but none of the other main effects or interactions was significant. Individual mean comparisons ( $t$ test) revealed that the interaction resulted from the significantly low performance on long probe trials. Accuracy on short and long nonprobe and short probe trials was equivalent and was significantly higher than on long probe trials. Also, accuracy on long probe trials was significantly below chance ( $t$ tests).

The results indicate that choice accuracy was systematically affected by signal modality. What is most interesting about the probe-trial deficit is that it is very similar to the "choose-short" effect (Spetch \& Wilkie, 1983). It has been reliably demonstrated that matching accuracy with duration samples is systematically biased when subjects are tested with long retention intervals. Under these conditions, subjects often express a strong tendency to choose the short-duration alternative following a longduration sample (Church, 1980; Spetch, 1987; Spetch \& Wilkie, 1983). The same choice bias, however, has appeared when duration samples are omitted entirely and the subject is merely presented with the choice alternatives (Church, 1980; Kraemer et al., 1985; Spetch \& Wilkie, 1983). The present results indicate yet another instance of a choose-short bias-when presented with unexpected choice stimuli, pigeons show a response bias for the stimulus associated with a short-duration sample. This result indicates that the birds had not learned to respond according to sample duration alone; if they had been relying only on sample duration, then accuracy would have been the same on probe and nonprobe trials. That this pattern did not change over the course of testing also suggests that the probe-trial deficit was not due to some type of nonspecific processing error, perhaps associated with a general change in test conditions from those of original training.

It should also be appreciated that these results contrast with studies that have found cross-modal transfer of conditional duration discriminations. For example, W. A. 
Roberts et al. (1989) found evidence that pigeons can be quite good at abstracting the duration of a sample independent of its modality. One important difference between the Roberts et al. study and the current experiment is that in the former, the same durations of light and tone samples were associated with the same set of choice stimuli, whereas in the latter, each sample modality was associated with a different set of choice stimuli. Thus, perhaps under some conditions, pigeons can be discouraged from relying exclusively on amodal attributes of a sample to select a choice alternative. It is also plausible that other procedural factors, which will be discussed later, could have produced differences in the results of the two experiments.

\section{EXPERIMENT 2}

The purpose of Experiment 2 was to assess the generality of the effect discovered in Experiment 1. A group of naive pigeons was again trained on two duration-SMTS problems, but unlike the first experiment, both problems involved visual signals. One problem involved mapping 2 - and 10-sec durations of a white ceiling light onto a set of color choice stimuli; the other problem involved mapping 2- and 10-sec durations of a red lamp, mounted on the front wall of the test chamber, onto a different set of color choices. The same general probe procedure used in Experiment 1 was then followed.

\section{Method}

Four naive adult White Carneaux pigeons were subjected to the same general training procedure that was used in the previous experiment, except that the order in which the two problems were acquired was counterbalanced. The two sample types consisted of a white ceiling light (W) and a red light presented from between the two side keys (R). The durations were again 2 and $10 \mathrm{sec}$. The correlation between sample type and correct choice stimulus for each bird can be discerned from Table 1 by simply substituting a new set of subject numbers and $R$ for the tone and $W$ for the light. The remaining aspects of the procedure followed exactly that described in the preceding experiment.

\section{Results and Discussion}

Trials to criterion on both problems are presented in Table 3. These scores were subjected to a mixed factorial ANOVA, with group (defined by which problem was acquired first) as the between-subjects factor and sample type (W or R) as the within-subjects factor. Although there was no overall difference in the rates at which the two problems were learned, there was a significant interaction between sample type and group $[F(1,2)=263.0$,

Table 3

Trials to Criterion in Experiment 2

\begin{tabular}{ccccc}
\hline Subject & Problem 1 & Trials & Problem 2 & Trials \\
\hline 9 & W & 74 & R & 19 \\
10 & W & 60 & R & 20 \\
11 & R & 44 & W & 9 \\
12 & R & 53 & W & 10 \\
\hline
\end{tabular}

Note $-\mathrm{W}=$ white ceiling light; $R=$ red wall light.
Table 4

Percent Correct on Nonprobe and Probe Trials in Experiment 2

\begin{tabular}{|c|c|c|c|}
\hline \multicolumn{2}{|c|}{ Nonprobe Trials } & \multicolumn{2}{|c|}{ Probe Trials } \\
\hline Short & Long & Short & Long \\
\hline 92 & 88 & 85 & 42 \\
\hline
\end{tabular}

$p<.05]$. The interaction indicates that subjects did better at acquiring their second problem than their first, regardless of the sample type.

The mean percent correct choices on probe and nonprobe test trials were subjected to a group $\times$ trial type $x$ signal type $x$ test block mixed ANOVA. This analysis confirmed that accuracy remained high on nonprobe trials $(M=89.6 \%)$ and was significantly higher than on probe trials $(M=59.9 \%)[F(1,2)=38.4]$. Also, this pattern of performance did not change over the four blocks of testing, and accuracy was equivalent for the two signal types, as indicated by the absence of significant testblock and signal-type main effects. Once again, however, it can be seen in Table 4 that performance on long and short samples was different on probe and nonprobe trials, although the interaction between trial type and sample duration did not quite reach significance $[F(1,2)=17.4$, $p<.07]$. Nonetheless, it can be seen that accuracy on long probe trials diminished to a near chance level (a $t$ test revealed that accuracy was not significantly below chance), whereas performance on the other trial types remained high. It should also be mentioned that the absence of a significant trial type $\times$ duration interaction could reflect inadequate statistical power emanating from the small sample size.

As in Experiment 1, pigeons were affected by the sensory-specific features of the sample and showed a response bias similar to the choose-short effect. On probe trials, they tended to select the choice alternative for a short-duration sample after a long-duration sample had been presented. The robustness of this effect can be inferred from the absence of any change in the effect over the course of testing, which again suggests that it is unlikely that the disruption on probe trials reflects some general performance deficit. As in the first experiment, it appears that samples were not processed solely on the basis of their duration; to select a choice alternative, the pigeons seem to have combined sensory-specific features of the sample with duration information.

These results contrast with evidence from Spetch and Sinha (1989), in which pigeons seemed to treat as the same signal distinctly different visual signals that were presented for the same duration. Two major differences exist between the two experiments: First, birds in the present experiment were trained with unique choice alternatives for each signal type, whereas Spetch and Sinha trained their pigeons with the same set of choice alternatives for both signal types. Another difference is that Spetch and Sinha presented a test in which two samples appeared in succession, and they based their conclusions on the tendency with which their pigeons added durations of the two sam- 
ples. In the present experiment, a probe-trial procedure was used that assessed the pigeon's ability to extrapolate duration information to a set of choice alternatives not previously associated with a given sample.

\section{GENERAL DISCUSSION}

The combined results of the two experiments indicate that pigeons do not necessarily ignore stimulus-specific attributes of a sample when processing signal duration, even when duration is the only informative cue. Pigeons were originally trained to select a different choice stimulus contingent on the duration of signals that varied with respect to either modality (Experiment 1) or visual characteristics (Experiment 2). A unique choice stimulus was associated with each combination of duration and signal type. This type of training seemed to prevent the birds from processing samples exclusively with respect to duration, despite the fact that they could have selected correct choices by relying on sample duration alone. More important, the modality or visual characteristics of the sample did not impart information about which of the two test stimuli was correct.

The results contrast with other findings that indicate that animals can functionally equate durations of different signal types. Cross-modal transfer of a duration discrimination has been found both in rats (Church \& Meck, 1984; S. Roberts, 1982) and pigeons (W. A. Roberts et al., 1989), and cross-dimensional transfer has appeared with pigeons (Spetch \& Sinha, 1989). One critical difference between these studies and the present experiments is that animals in the former were trained with the same response alternatives for different signal types, whereas pigeons in the latter were trained with different sets of response alternatives for each signal type tested. Thus, perhaps cross-modal or cross-dimensional transfer can be constrained by the structure of the training episode, and the tendency of an animal to select invariant properties of stimuli may not be based solely on the comparative relevance of those attributes; although sample duration was informative in the present experiments, modality or visual properties were not.

There is another important procedural feature of the present experiments that should be mentioned. A probe procedure was used to test cross-modal and crossdimensional processing of sample duration. In transferof-training studies, the performance unit is usually an entire session, and transfer is measured with respect to the mean accuracy for each session. The argument could be made that we presented an insufficient number of test trials and had we included more probe trials or continued testing for more sessions, a different pattern of results would have emerged. We disagree with this hypothesis for three reasons: First, the total number of probe trials we used was nearly the same as the number of trials that would be presented in one session of a typical transfer-of-training study. For example, W. A. Roberts et al. (1989) found evidence of cross-modal transfer in pigeons within the first transfer session, which contained nearly the same number of trials as we presented over the course of probetrial testing. Second, performance in Experiments 1 and 2 did not change over test blocks, indicating that there was no evidence that the pattern of performance that appeared on the first probe-trial session changed over the course of subsequent test sessions. Third, it is important to emphasize that a question different from those that have been considered in the transfer-of-training studies was under examination in the current study. We were interested in immediate transfer (i.e., how well pigeons could use what they had already learned) rather than general transfer (i.e., how quickly pigeons could learn), and therefore the probe procedure was appropriate and adequate (Holt \& Kehoe, 1985).

Church and Meck (1984) have shown that even when rats express cross-modal transfer, their performance is still somewhat influenced by stimulus-specific attributes. Thus, we would not conclude that the use of the same response alternatives prevents the use of stimulus-specific information but rather that the degree to which such information affects sample processing may be partially determined by the nature of the response rules involved. Just how multiple response sets might come to exert such an effect can only be speculated. One possibility is that the use of multiple sets of choice stimuli encourages some form of sample categorization, which induces the subject to differentiate samples according to signal type, as well as duration. The nature of the relationship between samples and choice stimuli has been shown to influence the tendency of pigeons to categorize or "commonly code", samples (Grant, 1982; Santi \& Roberts, 1985; Urcuioli, Zentall, Jackson-Smith, \& Steirn, 1989; Zentall, Urcuioli, Jagielo, \& Jackson-Smith, 1989). In transfer studies that have maintained the same set of response alternatives for two different signal types, the sample-response relationship has been like a many-to-one arrangement-each correct choice alternative has been associated with two different sample types of the same duration. The many-to-one arrangement may encourage a common coding process whereby nominally distinctive samples, as distinguished by all of their attributes, are functionally equated and treated as the same (Urcuioli et al., 1989; Zentall et al., 1989). Thus, it is possible that when the same choice stimuli are associated with the same durations of two different signal types, as in the W. A. Roberts et al. (1989) study, then equal durations of physically different signals will be coded as equivalent. When, however, different choice stimuli are associated with the same durations of different signal types, as was the case in the current experiments, common coding may not occur. Whether or not common coding occurs may determine whether or not immediate transfer appears.

Another phenomenon that may be relevant to the present findings is the miscuing effect that is found with one-tomany matching. Pigeons trained to match the same sample to two different choice stimuli often express performance deficits when tested with choice stimuli that are 
not anticipated. For example, birds might be trained to match a red sample to both a vertical line and a circle, and a green sample to both a horizontal line and a square. Half of the presentations of each sample would be followed by lines, and shapes would appear on the remaining trials. In addition, each set of choice stimuli would be preceded by a different cue (e.g., houselight before lines and tone before shapes). Under these conditions, the cue can influence choice accuracy, as indicated by performance when the choice stimuli are miscued. When the choice stimuli are not those associated with the preceding cue, accuracy declines (Grant \& MacDonald, 1990; Santi, Musgrave, \& Bradford, 1988; Stonebraker \& Rilling, 1984). If it is assumed that sample duration is the functional stimulus used to select an appropriate choice stimulus, then the probe procedure used here is very similar to the miscuing procedure. Accordingly, short and long sample durations, irrespective of signal type, might have been associated with two different choice stimuli, and the two sets of choice stimuli might have been cued by other relevant characteristics of the sample (e.g., modality in Experiment 1 and nature of the light in Experiment 2). The deficits found on probe trials could then be attributed to the adverse effects of miscuing. The logic presented by Grant \& MacDonald (1990) would further imply that the present results are consistent with a prospective-coding account of miscuing (Stonebraker \& Rilling, 1984). Performance on probe trials might indicate that an appropriate prospective code was unavailable, which raises another important issue.

There have been several alternative theoretical accounts of how pigeons code trials of duration SMTS. These alternatives differ with respect to the assumptions they make concerning what information is represented and how that information is coded. The analog-coding model adopts a retrospective view of memory processing and assumes that actual sample durations are encoded and remembered on each trial (Spetch \& Sinha, 1989; Spetch \& Wilkie, 1983). The categorical-coding model likewise adopts a retrospective view but assumes that sample durations are coded as relative categories, such as short and long (Church, 1980). The behavioral-mediation model avoids reference to any type of memorial code and instead assumes that trials are coded as differential behaviors correlated with different sample durations (Killeen \& Fetterman, 1988). Finally, the prospective-coding model assumes that memorial representation is involved but assumes that subjects code each trial with respect to the choice stimuli with which the duration samples are associated (Kraemer, Mazmanian, \& Roberts, 1985).

Each of these models has supporting evidence, as well as deficiencies (Parker \& Glover, 1987; Spetch, 1987; Spetch \& Rusak, 1989; Wilkie \& Willson, 1990). Although the current results provide no resolution, they may contribute to the debate. For example, the results from both experiments are quite consistent with a prospective- coding model, which assumes that each sample, uniquely defined by all of its attributes (e.g., duration, modality, location, intensity, etc.) is coded with respect to an appropriate choice stimulus. Following a duration of one signal type, the subject is ready to respond to a specific choice alternative and is unprepared when the available choices are those appropriate to the signal type that was not presented. The asymmetrical deficits found in both experiments, in which accuracy on short durations remained high and accuracy on long durations dropped substantially (below chance in Experiment 1), is consistent with the possibility that in the absence of an appropriate memory, a response bias appears for the stimulus correlated with a short-duration sample. This hypothesis is supported by evidence that a response bias appears when the duration samples are omitted (Church, 1980; Kraemer et al., 1985; Spetch \& Wilkie, 1983) and when nondurational samples are involved (Fetterman \& MacEwen, 1989).

Alternative models are far less effective in dealing with the present results. Minimally, some additional assumptions about how pigeons represent trials of duration SMTS would be required beyond those contained in current instantiations of these models. In contrast, the advantage of a prospective-coding model is that it is already sufficient to capture the present results, although it may have difficulty with other findings (Wilkie \& Willson, 1990).

\section{REFERENCES}

Church, R. M. (1978). The internal clock. In S. Hulse, H. Fowler, \& W. Honig (Eds.), Cognitive processes in animal behavior (pp. 277310). Hillsdale, NJ: Erlbaum.

Church, R. M. (1980). Short-term memory for time intervals. Learning \& Motivation, 11, 208-219.

Church, R. M., Meck, W. H. (1984). Acquisition and cross-modal transfer of classification rules for temporal intervals. In M. Commons, A. Wagner, \& R. Hernstein (Eds.), Quantitative analyses of behavior discriminative processes (Vol. 4, pp. 75-97). Cambridge, MA: Ballinger.

Delong, R. E., * Wasserman, E. A. (1985). Stimulus selection with duration as a relevant cue. Learning \& Motivation, 16, 259-267.

Fetterman, J. G., MacEwen, D. (1989). Short-term memory for responses: The "choose-small" effect. Joumal of the Experimental Analysis of Behavior, 52, 311-324

Gibbon, J., a Alan, L. (Eds.) (1984). Timing and time perception. New York: New York Academy of Sciences.

Grant, D. S. (1982). Prospective versus retrospective coding of samples of stimuli, responses, and reinforcers in delayed matching-tosample by pigeons. Learning \& Motivation, 13, 265-280.

Grant, D. S., \& MacDonald, S. E. (1990). An evaluation of the role of dual coding in mediating the effect of incorrectly cuing the comparison dimension in delayed matching in pigeons. Animal Leaming \& Behavior, 18, 151-156.

Holt, P. E., Kenoe, E. J. (1985). Cross-modal transfer as a function of similarities between training tasks in classical conditioning of the rabbit. Animal Learning \& Behavior, 13, 51-59.

Killeen, P. R., \& FetTerman, J. G. (1988). A behavioral theory of timing. Psychological Review, 95, 274-295.

Kraemer, P. J., Mazmanian, D. S., Roberts, W. A. (1985). The choose-short effect in pigeon memory for stimulus duration: Subjective shortening versus coding models. Animal Learning \& Behavior, 13, 349-354. 
Kraemer, P. J., Roberts, W. A. (1984). Short-term memory for visual and auditory stimuli in pigeons. Animal Learning \& Behavior, 12, 275-284.

PARKer, B. K., Glover, R. L. (1987). Event duration memory: The effects of delay-interval illumination and instructional cuing. Animal Learning \& Behavior, 15, 241-248.

Richelle, M., \& LejeUne, H. (1990). Time in animal behavior. OXford, U.K.: Pergamon Press.

ROBERTS, S. (1982). Cross-modal use of an internal clock. Journal of Experimental Psychology: Animal Behavior Processes, 8, 2-22.

Roberts, W. A., Cheng, K., \& Cohen, J. S. (1989). Timing light and tone signals in pigeons. Joumal of Experimental Psychology: Animal Behavior Processes, 15, 23-35.

Santi, A. Musgrave, S., \& Bradford, S. A. (1988). Utilization of cues signaling different test stimulus dimensions in delayed matching to sample by pigeons. Learning \& Motivation, 19, 87-98.

SANTI, A., Roberts, W. A. (1985). Prospective representation: The effects of varied mapping of sample stimuli to comparison stimuli and differential trial outcome on pigeons' working memory. Animal Learning \& Behavior, 13, 103-108

SPETCH, M. L. (1987). Systematic errors in pigeons' memory for event duration: Interaction between training and test delay. Animal Learning \& Behavior, 15, 1-5.

SPETCH, M. L., \& RuSAK, B. (1989). Pigeons' memory for event duration: Intertrial interval and delay effects. Animal Leaming \& Behavior, 17, 147-156.
SPETch, M. L., Sinha, S. S. (1989). Proactive effects in pigeons' memory for event duration: Evidence for analogical retention. Journal of Experimental Psychology: Animal Behavior Processes, 15, 347-357.

SPETCH, M. L., WILKIE, D. M. (1983). Subjective shortening: A model of pigeons' memory for event duration. Joumal of Experimental Psychology: Animal Behavior Processes, 9, 14-30.

Stonebraker, T. B., \& Rilling, M. (1984). Retrospective versus prospective processes in delayed matching to sample. Bulletin of the Psychonomic Society, 22, 372-375.

Urcuiol, P. J., Zentall, T. R., Jackson-Smith, P., \& Steirn, J. N. (1989). Evidence for common coding in many-to-one-matching: Retention, intertrial interference, and transfer. Jourmal of Experimental Psychology: Animal Behavior Processes, 15, 264-273.

WILKIE, D. M., WILlson, R. J. (1990). Discriminal distance analysis supports the hypothesis that pigeons retrospectively encode event duration. Animal Learning \& Behavior, 18, 124-132.

Zentall, T. R., Urcuiol, P. J., Jagielo, J. A., \& Jackson-Smith, P. (1989). Interaction of sample dimension and sample-comparison mapping on pigeons' performance of delayed conditional discriminations. Animal Learning \& Behavior, 17, 172-178.

(Manuscript received June 26, 1990; revision accepted for publication February 21, 1991.) 